\title{
UMA NOVA METODOLOGIA PARA FLUXOS DE CARGA FUZZY
}

\author{
Marcelo Murad Pereira Lima* \\ murad@zipmail.com.br
}

\author{
Marcelo Adorni Pereira* \\ adorni@altavista.net
}

*UNICAMP-FEEC-DSEE, Caixa Postal 6101, 13081-970, Campinas, São Paulo, BRASIL

\author{
Carlos Alberto Favarin Murari* \\ murari@dsee.fee.unicamp.br
}

quando comparados com outros métodos e que podem ser interpretados através de termos lingüisticos.

PALAVRAS-CHAVE: Conjuntos nebulosos, fluxo de carga.

\section{NOMENCLATURA}

FCN - Fluxo de Carga Newton

FCL - Fluxo de Carga Linearizado

FCNTrap - Fluxo de Carga Newton fuzzy proposto em Miranda et alli. (1990)

FCLTrap - Fluxo de Carga Linearizado proposto em Miranda et alli. (1990)

$B$ - Matriz utilizada no cálculo do FCL (Monticelli, A. (1983))

$J$ - Matriz Jacobiana do FCN

$\Omega_{k} \quad$-Conjunto das barras conectadas à barra $k$

$K$ - Conjunto das barras conectadas à barra $k$ mais a própria barra $k$

$\tilde{X}$ - Variável na forma fuzzy

$\oplus, \Theta$ e $\otimes$ - Adição, Subtração e Multiplicação fuzzy (Pereira, M. A. \& Murari, C. A. F., 1999)

Q - Multiplicação fuzzy modificada (Apêndice A)

$\underline{\cos }$ e $\underline{\sin }$ - Cosseno e Seno fuzzy (Apêndice B)

\section{INTRODUÇÃO}

Nas fases de modelagem dos sistemas elétricos de potência e adequação das variáveis presentes nos sistemas reais, informações relativas aos conhecimentos e experiências de técnicos e engenheiros não podiam ser utiliza- 
das integralmente até algum tempo atrás. Informações do tipo 'o fluxo na linha $\mathrm{km}$ está alto' dificilmente poderiam ser quantificadas e empregadas em um algoritmo computacional que implicasse em uma decisão imediata.

Os fluxos de carga determinísticos ou probabilísticos não eram capazes de representar tais informações (Kenarangui, R. \& Seifi, A., 1994) integralmente, já que os determinísticos consideram os dados como constantes e os probabilísticos são baseados em repetições de eventos ou em dados experimentais.

Os fluxos de carga probabilísticos ainda apresentam a desvantagem de necessitarem de rotinas de cálculo mais complexas (Meliopoulos et alli., 1990).

Em muitos casos, as incertezas presentes nos sistemas eram ignoradas, ficando sem alternativas para observações ou análises do tipo 'se a tensão na barra $k$ é muito baixa, desligar a carga nesta barra'.

A teoria de conjuntos nebulosos (Zadeh, L. A., 1965) e a teoria de possibilidades (Zadeh, L. A., 1978) desenvolvidas por Lofti Zadeh viabilizaram o desenvolvimento de fluxos de carga como em Miranda et alli. (1990) e Kenarangui, R. \& Seifi, A. (1994). Nestes artigos são apresentados fluxos de carga que utilizam funções trapezoidais para a representação das variáveis do problema. Para efetuar os cálculos, os autores aplicam linearizações baseadas em série de Taylor, matemática intervalar e "alfa cortes" (Pedrycz, W. \& Gomide, F., 1998).

Neste trabalho é proposto um fluxo de carga capaz de, a partir de dados de entrada (cargas, gerações, etc.) com imprecisões e utilizando operações matemáticas nebulosas, fornecer como resultados números nebulosos, utilizando-se funções de pertinência sinusoidais.

\section{REPRESENTAC̄̃̃O DOS DADOS}

Como alternativa aos métodos Miranda et alli. (1990) e Kenarangui, R. \& Seifi, A. (1994) que utilizam funções trapezoidais (figura 1) para os números nebulosos, este trabalho utiliza uma função sinusoidal conforme figura 2 e equação (1). Este tipo de representação possibilitou o desenvolvimento de operações algébricas de fácil implementação computacional e cujos dados e resultados são representados por apenas dois parâmetros.

$$
\tilde{A}=A(x)=\frac{1}{1+\left(\frac{x-m_{A}}{\alpha_{A}}\right)^{2}}
$$

A equação (1) também é definida por $A(m, \alpha)$, onde $m$

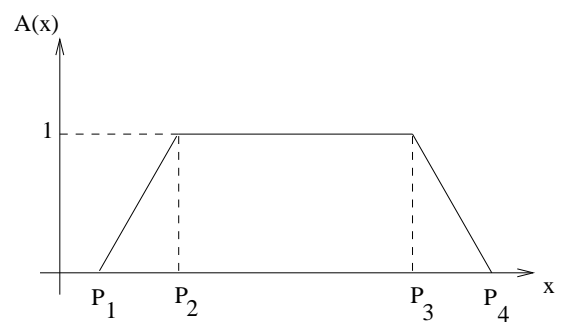

Figura 1: Função trapezoidal.

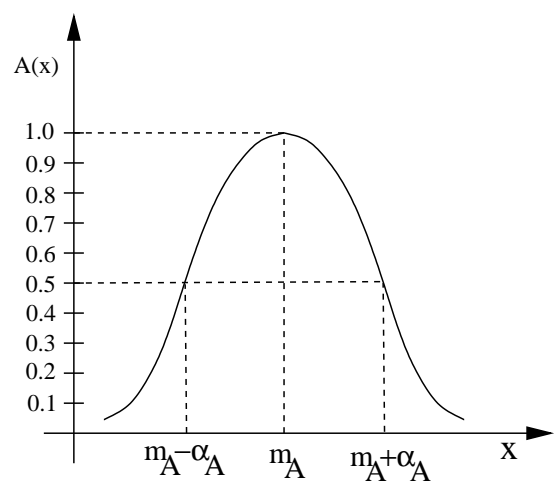

Figura 2: Função em forma de sino (Sinusoidal)

corresponde ao valor de maior grau, ou seja, $A(m)=1$, e o parâmetro $\alpha$ é o grau de incerteza da função, sendo $A(m+\alpha)=A(m-\alpha)=0,5$.

As imprecisões são atribuídas apenas aos dados de potência ativa e reativa. No banco de dados, os valores de potência ativa e reativa correspondem ao parâmetro $m$ e o parâmetro $\alpha$ define uma determinada imprecisão, que nesta aplicação foi fixada em $5 \%$ de $m$. Para a função trapezoidal os pontos $\mathrm{P}_{1}, \mathrm{P}_{2}, \mathrm{P}_{3}$ e $\mathrm{P}_{4}$ correspondem aos pontos $0,93 . m, 0,95 . m, 1,05 . m$ e $1,07 . m$ respectivamente.

\section{DESENVOLVIMENTO DOS FLUXOS DE CARGA FUZZY}

Os fluxos de carga fuzzy são fundamentados nos fluxos de carga convencionais: FCN e FCL (Monticelli, A., 1983), onde algumas operações matemáticas foram substituídas por operações nebulosas.

\subsection{Fluxo de Carga Linearizado Fuzzy - FCLF}

O FCLF é simulado em duas iterações para que se possa incluir as perdas de potência ativa e assim obter uma melhor estimativa para os ângulos. 
Procedimento:

- A partir das potências nas barras $(\tilde{P})$ resolver o sistema matricial:

$$
[\tilde{\theta}]=[B]^{-1} \otimes[\tilde{P}]
$$

- De posse desta primeira estimativa para os ângulos, calcular as perdas nas ligações:

$$
\tilde{P}_{k m}^{\text {Perdas }}=\tilde{\theta}_{k m}^{2} \otimes g_{k m}
$$

- Adicionar metade dos valores das perdas nas respectivas barras terminais e obter um novo vetor $\tilde{P}$ que é aplicado no sistema matricial da equação (2), obtendo-se os valores finais para os ângulos.

- Calcular os fluxos de potência ativa nas linhas:

$$
\tilde{P}_{k m}=\sum_{m \in \Omega_{k}} x_{k m}^{-1} \otimes \tilde{\theta}_{k m}
$$

Com isto tem-se como resultado as variáveis de saída do FCL na forma de números nebulosos, conforme equação (1).

\subsection{Fluxo de Carga Newton Fuzzy - FCNF}

O FCNF foi desenvolvido da seguinte maneira:

- Com base no ponto de maior grau $(m)$ dos dados de entrada, resolver um FCN convencional, obtendo-se os valores dos ângulos e das magnitudes das tensões, que serão os valores correspondentes aos pontos centrais $(m)$ das distribuições de possibilidade para estas variáveis.

- Para determinar os valores dos desvios $(\alpha)$ destas variáveis, utilizar a matriz Jacobiana obtida na última iteração do FCN, seguindo a equação (5), onde o vetor $\underline{\alpha}_{L I Q P Q}$ é formado pelos valores de alfa de $\tilde{P}\left(m_{P}, \alpha_{P}\right)$ e $\tilde{Q}\left(m_{Q}, \alpha_{Q}\right)$, fornecidos nos dados iniciais.

$$
\left[\alpha_{\theta v}\right]=[J]^{-1} *\left[\alpha_{L I Q P Q}\right]
$$

- De posse das distribuições de possibilidade para os ângulos e magnitudes das tensões, calcular as po- tências geradas nas barras (equação (6)).

$$
\begin{gathered}
\tilde{P}_{k}=\tilde{V}_{k} \sum_{m \in K} \tilde{V}_{m} \otimes\left[G_{k m} \otimes \underline{\cos }\left(\tilde{\theta}_{k m} \oplus \varphi_{k m}\right) \oplus\right. \\
\left.\oplus B_{k m} \otimes \underline{\sin }\left(\tilde{\theta}_{k m} \oplus \varphi_{k m}\right)\right] \\
\tilde{Q}_{k}=\tilde{V}_{k} \sum_{m \in K} \tilde{V}_{m} \otimes\left[G_{k m} \otimes \underline{\sin }\left(\tilde{\theta}_{k m} \oplus \varphi_{k m}\right) \Theta\right. \\
\left.\Theta B_{k m} \otimes \underline{\cos }\left(\tilde{\theta}_{k m} \oplus \varphi_{k m}\right)\right]
\end{gathered}
$$

- Calcular os fluxos:

$$
\begin{aligned}
\tilde{P}_{k m}= & g_{k m} \underline{\otimes} \tilde{V}_{k}^{2} \Theta t^{2 a p_{k m}} \underline{\otimes} \tilde{V}_{k} \underline{\otimes} \tilde{V}_{m} \underline{\otimes} \\
& \underline{\otimes}\left[g_{k m} \underline{\otimes} \underline{\cos }\left(\tilde{\theta}_{k m} \oplus \varphi_{k m}\right) \oplus b_{k m} \underline{\otimes} \underline{\sin }\left(\tilde{\theta}_{k m} \oplus \varphi_{k m}\right)\right] \\
\tilde{P}_{m k}= & g_{k m} \underline{\otimes}\left(\operatorname{tap}_{k m} \underline{\otimes} \tilde{V}_{m}\right)^{2} \Theta t a p_{k m} \underline{\otimes} \tilde{V}_{k} \underline{\otimes} \tilde{V}_{m} \underline{\otimes} \\
& \underline{\otimes}\left[g_{k m} \underline{\otimes} \underline{\cos }\left(\tilde{\theta}_{k m} \oplus \varphi_{k m}\right) \Theta b_{k m} \underline{\otimes} \underline{\sin }\left(\tilde{\theta}_{k m} \oplus \varphi_{k m}\right)\right]
\end{aligned}
$$

$$
\begin{aligned}
\tilde{Q}_{k m}= & -\left(b_{k m}+b_{k}^{\text {shunt }}\right) \underline{\otimes} \tilde{V}_{k}^{2} \oplus \operatorname{tap} p_{k m} \underline{\otimes} \tilde{V}_{k} \underline{\otimes} \tilde{V}_{m} \underline{\otimes} \\
\underline{\otimes} & {\left[b_{k m} \underline{\otimes} \underline{\cos }\left(\tilde{\theta}_{k m} \oplus \varphi_{k m}\right) \Theta g_{k m} \underline{\otimes} \underline{\sin }\left(\tilde{\theta}_{k m} \oplus \varphi_{k m}\right)\right] } \\
\tilde{Q}_{m k}= & -\left(b_{k m}+b_{k}^{\text {shunt }}\right) \underline{\otimes}\left(\operatorname{tap}_{k m} \underline{\otimes} \tilde{V}_{m}\right)^{2} \oplus t a p_{k m} \underline{\otimes} \\
& \underline{\otimes} \tilde{V}_{k} \underline{\otimes} \tilde{V}_{m} \underline{\otimes}\left[b_{k m} \underline{\otimes} \underline{\cos }\left(\tilde{\theta}_{k m} \oplus \varphi_{k m}\right) \oplus g_{k m} \underline{\otimes}\right. \\
& \left.\underline{\otimes} \underline{\sin }\left(\tilde{\theta}_{k m} \oplus \varphi_{k m}\right)\right]
\end{aligned}
$$

- Calcular as perdas:

$$
\begin{aligned}
\tilde{P}_{k m}^{\text {Perdas }}= & g_{k m} \otimes \tilde{V}_{k}^{2} \oplus g_{k m} \otimes\left(\operatorname{tap}_{k m} \otimes \tilde{V}_{m}\right)^{2} \Theta \\
& \Theta 2 \otimes \tilde{V}_{k} \otimes \tilde{V}_{m} g_{k m} \otimes \underline{\cos }\left(\tilde{\theta}_{k m} \oplus \varphi_{k m}\right)
\end{aligned}
$$

Observe que as equações (6) e (9) utilizam o multiplicador nebuloso de Pereira, M. A. \& Murari, C. A. F. (1999) e as equações (7) e (8), o multiplicador do Apêndice $\mathrm{A}$.

Com estes valores pode-se obter as distribuições de possibilidade das variáveis de saída. Fez-se necessária a utilização de dois tipos de função de multiplicação pois algumas expressões utilizadas nos cálculos possuem termos com muitas multiplicações e como as operações com números nebulosos não são tão "comportadas" como as da matemática convencional (Pedrycz, W \& Gomide, F., 1998) eram obtidos valores para os desvios não compatíveis com os esperados. 


\section{SIMULAÇÃO}

As tabelas apresentadas nesta seção contém resultados obtidos para as versões de fluxo de carga fuzzy aplicadas ao sistema IEEE30 (Freris, L. L. \& Sasson, A. M., 1968).

Os resultados obtidos com FCLF e FCNF, correspondentes aos pontos $m$ - $\alpha$ e $m+\alpha$, são comparados com os resultados obtidos com FCL e FCN aplicados em duas etapas, uma com os dados para a potência $P_{\text {min }}=m_{p^{-}}$ $\alpha_{p}, Q_{\min }=m_{Q}-\alpha_{Q}$ e outra para a potência $P_{\max }=$ $m_{p}+\alpha_{p}, Q_{\max }=m_{Q}+\alpha_{Q}$.

Estes pontos também correspondem a $P_{2}$ e $P_{3}$ na função trapezoidal, respectivamente.

$\mathrm{Na}$ impossibilidade de incluir todos os resultados, as tabelas apresentam parte deles.

\subsection{Resultados com o FCLF}

A tabela 1 apresenta os valores dos ângulos das tensões nodais.

Tabela 1: Ângulos (graus)

\begin{tabular}{|c|c|c|c|c|c|c|}
\hline Barra & \multicolumn{2}{|c|}{ FCL } & \multicolumn{2}{c|}{ FCLF } & \multicolumn{2}{c|}{ FCLTrap } \\
\hline $\mathrm{N}^{0}$ & $\theta_{P \min }$ & $\theta_{P \max }$ & $\theta_{(m-\alpha)}$ & $\theta_{(m+\alpha)}$ & $\theta_{P 2}$ & $\theta_{P 3}$ \\
\hline 2 & $-5,44$ & $-6,04$ & $-5,39$ & $-6,10$ & $-5,41$ & $-6,06$ \\
\hline 3 & $-8,16$ & $-9,05$ & $-8,12$ & $-9,08$ & $-8,15$ & $-9,04$ \\
\hline 4 & $-9,74$ & $-10,8$ & $-9,70$ & $-10,9$ & $-9,74$ & $-10,8$ \\
\hline 5 & $-14,1$ & $-15,6$ & $-14,0$ & $-15,7$ & $-14,0$ & $-15,6$ \\
\hline 11 & $-14,6$ & $-16,1$ & $-14,5$ & $-16,2$ & $-14,5$ & $-16,2$ \\
\hline 12 & $-15,6$ & $-17,3$ & $-15,5$ & $-17,3$ & $-15,7$ & $-17,2$ \\
\hline 13 & $-15,6$ & $-17,3$ & $-15,5$ & $-17,3$ & $-15,5$ & $-17,2$ \\
\hline 14 & $-16,6$ & $-18,4$ & $-16,6$ & $-18,5$ & $-16,6$ & $-18,4$ \\
\hline 29 & $-17,6$ & $-19,5$ & $-17,5$ & $-19,5$ & $-17,5$ & $-19,4$ \\
\hline 30 & $-18,5$ & $-20,5$ & $-18,4$ & $-20,5$ & $-18,4$ & $-20,4$ \\
\hline
\end{tabular}

A tabela 2 apresenta os erros percentuais dos ângulos obtidos pelo FCLF e o proposto em Miranda et alli. (1990).

Tabela 2: Erro (\%) para os Ângulos

\begin{tabular}{|c|c|c|c|c|}
\hline Barra & \multicolumn{2}{|c|}{ FCLF } & \multicolumn{2}{c|}{ FCLTrap } \\
\hline $\mathrm{N}^{o}$ & $\theta_{(m-\alpha)}$ & $\theta_{(m+\alpha)}$ & $\theta_{P 2}$ & $\theta_{P 3}$ \\
\hline 2 & 1,04 & $-0,91$ & 2,57 & $-2,41$ \\
\hline 3 & 0,43 & $-0,36$ & 2,10 & $-1,97$ \\
\hline 4 & 0,43 & $-0,37$ & 2,13 & $-2,00$ \\
\hline 5 & 0,36 & $-0,31$ & 2,14 & $-2,01$ \\
\hline 11 & 0,31 & $-0,26$ & 2,10 & $-1,97$ \\
\hline 12 & 0,28 & $-0,24$ & 2,09 & $-1,96$ \\
\hline 13 & 0,28 & $-0,24$ & 2,09 & $-1,96$ \\
\hline 14 & 0,26 & $-0,22$ & 2,09 & $-1,96$ \\
\hline 29 & 0,25 & $-0,22$ & 2,08 & $-1,95$ \\
\hline 30 & 0,24 & $-0,21$ & 2,07 & $-1,94$ \\
\hline
\end{tabular}

Além dos erros percentuais correspondentes aos pontos $\mathrm{P}_{2}$ e $\mathrm{P}_{3}$ serem maiores que os de $\theta_{(m-\alpha)}$ e $\theta_{(m+\alpha)}$, a sua função de pertinência vale 1 (função trapezoidal (Miranda et alli., 1990)) enquanto que para $\theta_{(m-\alpha)} \mathrm{e}$ $\theta_{(m+\alpha)}$ vale 0,50 (figura 2 ).

A tabela 3 apresenta resultados para os fluxos de potência ativa nas linhas e a tabela 4 expõe os erros percentuais para o FCLF e o proposto em Miranda et alli. (1990).

Tabela 3: Fluxo de Potência Ativa (MW)

\begin{tabular}{|c|c|c|c|c|c|c|}
\hline Linha & \multicolumn{2}{|c|}{ FCL } & \multicolumn{2}{c|}{ FCLF } & \multicolumn{2}{c|}{ FCLTrap } \\
\hline Ni-Nf & $P_{k m}^{\text {min }}$ & $P_{k m}^{\text {max }}$ & $P_{k m}^{m-\alpha}$ & $P_{k m}^{m+\alpha}$ & $P_{k m}^{P 2}$ & $P_{k m}^{P 3}$ \\
\hline 12 & 165,2 & 183,5 & 163,5 & 185,2 & 164,5 & 184,1 \\
\hline 13 & 76,86 & 85,29 & 76,53 & 85,60 & 76,89 & 85,25 \\
\hline 24 & 43,22 & 47,87 & 43,37 & 47,72 & 43,32 & 47,78 \\
\hline 34 & 73,11 & 80,98 & 72,80 & 81,29 & 72,86 & 81,22 \\
\hline 1415 & 1,22 & 1,35 & 1,22 & 1,35 & 0,845 & 1,725 \\
\hline 1617 & 2,61 & 2,88 & 2,60 & 2,89 & 2,037 & 3,452 \\
\hline 1518 & 5,28 & 5,84 & 5,28 & 5,84 & 4,874 & 6,240 \\
\hline 1819 & 2,22 & 2,46 & 2,22 & 2,46 & 1,679 & 3,006 \\
\hline 2930 & 3,50 & 3,87 & 3,50 & 3,87 & 3,431 & 3,933 \\
\hline 828 & $-0,32$ & $-0,35$ & $-0,32$ & $-0,35$ & 0,084 & $-0,75$ \\
\hline
\end{tabular}

Tabela 4: Erro (\%) para Fluxos de Potência Ativa

\begin{tabular}{|c|c|c|c|c|}
\hline Linha & \multicolumn{2}{|c|}{ FCLF } & \multicolumn{2}{c|}{ FCLTrap } \\
\hline Ni-Nf & $P_{k m}^{m-\alpha}$ & $P_{k m}^{m+\alpha}$ & $P_{k m}^{P 2}$ & $P_{k m}^{P 3}$ \\
\hline 12 & 1,04 & $-2,98$ & 0,44 & $-0,34$ \\
\hline 13 & 0,43 & $-2,40$ & $-0,04$ & 0,05 \\
\hline 24 & $-0,35$ & $-1,65$ & $-0,22$ & 0,20 \\
\hline 34 & 0,43 & $-2,37$ & 0,34 & $-0,30$ \\
\hline 1415 & 0,10 & $-2,05$ & 30,75 & $-27,8$ \\
\hline 1617 & 0,17 & $-2,11$ & 21,97 & $-19,9$ \\
\hline 1518 & 0,05 & $-1,99$ & 7,70 & $-6,85$ \\
\hline 1819 & 0,11 & $-2,05$ & 24,37 & $-22,2$ \\
\hline 2930 & 0,00 & $-1,95$ & 1,97 & $-1,63$ \\
\hline 828 & 0,13 & $-1,88$ & -133 & 123,9 \\
\hline
\end{tabular}

Novamente o FCLF apresentou melhores resultados do que a metodologia proposta em Miranda et alli. (1990) pois o FCLF apresenta todos os erros inferiores a $3 \%$ o que não ocorre com o fluxo de carga proposto em Miranda et alli. (1990), alternando resultados bons e ruins (tabela 4).

No artigo Miranda et alli. (1990) não é proposta uma metodologia para o cálculo da potência gerada na barra de referência e para as perdas na transmissão. As tabelas 5 e 6 apresentam estes valores, obtidos com o FCLF, bem como os erros percentuais, comparados com o FCL.

Tabela 5: Potência Ativa Gerada (MW) e Erro

\begin{tabular}{|c|c|c|c|c|c|c|}
\hline Barra & \multicolumn{2}{|c|}{ FCL } & \multicolumn{2}{c|}{ FCLF } & \multicolumn{2}{c|}{ Erro \% } \\
\hline $\mathrm{N}^{o}$ & $P_{g}^{\min }$ & $P_{g}^{\max }$ & $P_{g}^{m-\alpha}$ & $P_{g}^{m+\alpha}$ & $P_{g}^{m-\alpha}$ & $P_{g}^{m+\alpha}$ \\
\hline 1 & 247,4 & 263,4 & 239,9 & 270,8 & 0,846 & $-0,74$ \\
\hline
\end{tabular}


Tabela 6: Perdas (MW) na Transmissão e Erros

\begin{tabular}{|c|c|c|c|c|c|c|}
\hline Linha & \multicolumn{2}{|c|}{ FCL } & \multicolumn{2}{c|}{ FCLF } & \multicolumn{2}{c|}{ Erro (\%) } \\
\hline Ni-Nf & $P_{\text {min }}^{\text {Per }}$ & $P_{m a x}^{\text {Per }}$ & $P_{m-\alpha}^{\text {Per }}$ & $P_{m+\alpha}^{\text {Per }}$ & $P_{m-\alpha}^{\text {Per }}$ & $P_{m+\alpha}^{\text {Per }}$ \\
\hline 12 & 2,14 & 2,61 & 2,307 & 2,940 & $-7,94$ & $-12,6$ \\
\hline 13 & 1,17 & 1,42 & 1,249 & 1,554 & $-7,22$ & $-9,18$ \\
\hline 24 & 0,46 & 0,56 & 0,484 & 0,583 & $-4,87$ & $-3,46$ \\
\hline 34 & 0,30 & 0,37 & 0,312 & 0,387 & $-3,28$ & $-4,85$ \\
\hline 1017 & 0,005 & 0,006 & 0,005 & 0,006 & 0 & 0 \\
\hline 1021 & 0,030 & 0,037 & 0,031 & 0,037 & $-0,99$ & 0 \\
\hline 1022 & 0,014 & 0,017 & 0,014 & 0,017 & 0 & 0 \\
\hline
\end{tabular}

O FCLF mostra ter boa precisão e utiliza uma função de pertinência de fácil implementação.

\subsection{Resultados com o FCNF}

Nas tabelas 7 e 8 são apresentados os resultados para as magnitudes e os ângulos das tensões obtidas a partir das simulações dos fluxos FCN, FCNF e FCNTrap.

Tabela 7: Magnitudes das Tensões (p.u.)

\begin{tabular}{|c|c|c|c|c|c|c|}
\hline Barra & \multicolumn{2}{|c|}{ FCN } & \multicolumn{2}{c|}{ FCNF } & \multicolumn{2}{c|}{ FCNTrap } \\
\hline $\mathrm{N}^{\circ}$ & $\mathrm{v}_{P \text { min }}$ & $\mathrm{v}_{P \max }$ & $\mathrm{v}_{(m-\alpha)}$ & $\mathrm{v}_{(m+\alpha)}$ & $\mathrm{v}_{P 2}$ & $\mathrm{v}_{P 3}$ \\
\hline 2 & 1,043 & 1,043 & 1,043 & 1,043 & 1,043 & 1,043 \\
\hline 3 & 1,023 & 1,021 & 1,023 & 1,021 & 1,023 & 1,021 \\
\hline 4 & 1,015 & 1,012 & 1,015 & 1,012 & 1,015 & 1,012 \\
\hline 5 & 1,010 & 1,010 & 1,010 & 1,010 & 1,010 & 1,010 \\
\hline 11 & 1,082 & 1,082 & 1,082 & 1,082 & 1,082 & 1,082 \\
\hline 12 & 1,058 & 1,055 & 1,058 & 1,055 & 1,058 & 1,055 \\
\hline 13 & 1,071 & 1,071 & 1,071 & 1,071 & 1,071 & 1,071 \\
\hline 14 & 1,045 & 1,039 & 1,045 & 1,039 & 1,045 & 1,039 \\
\hline 29 & 1,010 & 1,002 & 1,010 & 1,002 & 1,010 & 1,002 \\
\hline 30 & 0,999 & 0,990 & 0,999 & 0,990 & 1,000 & 0,990 \\
\hline
\end{tabular}

Tabela 8: Ângulos (graus)

\begin{tabular}{|c|c|c|c|c|c|c|}
\hline Barra & \multicolumn{2}{|c|}{ FCN } & \multicolumn{2}{c|}{ FCNF } & \multicolumn{2}{c|}{ FCNTrap } \\
\hline $\mathrm{N}^{\circ}$ & $\theta_{P \min }$ & $\theta_{P \max }$ & $\theta_{(m-\alpha)}$ & $\theta_{(m+\alpha)}$ & $\theta_{P 2}$ & $\theta_{P 3}$ \\
\hline 2 & $-5,25$ & $-5,75$ & $-5,25$ & $-5,75$ & $-5,14$ & $-5,86$ \\
\hline 3 & $-7,63$ & $-8,37$ & $-7,63$ & $-8,36$ & $-7,52$ & $-8,47$ \\
\hline 4 & $-9,21$ & $-10,1$ & $-9,21$ & $-10,1$ & $-9,08$ & $-10,2$ \\
\hline 5 & $-13,8$ & $-15,0$ & $-13,8$ & $-14,9$ & $-13,5$ & $-15,2$ \\
\hline 11 & $-14,1$ & $-15,5$ & $-14,1$ & $-15,5$ & $-13,9$ & $-15,6$ \\
\hline 12 & $-15,1$ & $-16,6$ & $-15,1$ & $-16,6$ & $-14,9$ & $-16,8$ \\
\hline 13 & $-15,1$ & $-16,6$ & $-15,1$ & $-16,6$ & $-14,9$ & $-16,8$ \\
\hline 14 & $-15,9$ & $-17,5$ & $-15,9$ & $-17,5$ & $-15,8$ & $-17,7$ \\
\hline 29 & $-16,6$ & $-18,3$ & $-16,6$ & $-18,3$ & $-16,4$ & $-18,5$ \\
\hline 30 & $-17,4$ & $-19,2$ & $-17,4$ & $-19,2$ & $-17,3$ & $-19,4$ \\
\hline
\end{tabular}

Os resultados para as magnitudes das tensões apresentam erros inferiores a $0,01 \%$ para o FCNF e também para o fluxo de carga proposto em Miranda et alli. (1990). Para os ângulos, a rotina de Miranda et alli. (1990) não apresenta resultados tão bons quanto para as magnitudes das tensões (erros de até 2,12\%), enquanto que o FCNF apresenta erros inferiores a $0,04 \%$. Na tabela 9 tem-se os resultados para a potência ativa gerada.

Nota-se que os resultados para o FCNF apresentam erros abaixo de $0,10 \%$ e para a rotina de Miranda et alli. (1990) os erros estão abaixo de 1,85\%. Na tabela 10
Tabela 9: Potência Ativa Gerada (MW)

\begin{tabular}{|c|c|c|c|c|c|c|}
\hline Barra & \multicolumn{2}{|c|}{ FCN } & \multicolumn{2}{c|}{ FCNF } & \multicolumn{2}{c|}{ FCNTrap } \\
\hline $\mathrm{N}^{o}$ & $P_{g}^{\text {min }}$ & $P_{g}^{\max }$ & $P_{g}^{m-\alpha}$ & $P_{g}^{m+\alpha}$ & $P_{g}^{P 2}$ & $P_{g}^{P 3}$ \\
\hline 1 & 249,5 & 272,6 & 249,5 & 272,5 & 249,7 & 272,3 \\
\hline
\end{tabular}

Tabela 11: Erro (\%) para Potência Reativa Gerada

\begin{tabular}{|c|c|c|c|c|}
\hline Barra & \multicolumn{2}{|c|}{ FCNF } & \multicolumn{2}{c|}{ FCNTrap } \\
\hline $\mathrm{N}^{o}$ & $Q_{g}^{m-\alpha}$ & $Q_{g}^{m+\alpha}$ & $Q_{g}^{P 2}$ & $Q_{g}^{P 3}$ \\
\hline 1 & 8,48 & $-7,17$ & 5,111 & $-4,64$ \\
\hline 2 & 3,42 & $-2,71$ & 4,073 & $-3,07$ \\
\hline 5 & $-6,41$ & 5,08 & 5,125 & $-3,81$ \\
\hline 8 & 0,33 & 2,16 & $-8,27$ & $-4,98$ \\
\hline 11 & 0,06 & 0,06 & 0,179 & $-0,05$ \\
\hline 13 & 0,14 & 0,12 & 0,316 & $-0,01$ \\
\hline
\end{tabular}

tem-se a potência reativa gerada e na tabela 11 os respectivos erros percentuais.

Tabela 10: Potência Reativa Gerada (MVAr)

\begin{tabular}{|c|c|c|c|c|c|c|}
\hline Barra & \multicolumn{2}{|c|}{ FCN } & \multicolumn{2}{c|}{ FCNF } & \multicolumn{2}{c|}{ FCNTrap } \\
\hline $\mathrm{N}^{\circ}$ & $Q_{g}^{\text {min }}$ & $Q_{g}^{\text {max }}$ & $Q_{g}^{m-\alpha}$ & $Q_{g}^{m+\alpha}$ & $Q_{g}^{P 2}$ & $Q_{g}^{P 3}$ \\
\hline 1 & $-15,8$ & $-18,8$ & $-14,4$ & $-20,1$ & $-14,9$ & $-19,6$ \\
\hline 2 & 32,47 & 39,10 & 31,36 & 40,16 & 31,14 & 40,30 \\
\hline 5 & 14,79 & 19,00 & 15,74 & 18,03 & 14,03 & 19,72 \\
\hline 8 & $-3,07$ & 3,67 & $-3,06$ & 3,59 & $-3,32$ & 3,85 \\
\hline 11 & 14,63 & 16,45 & 14,62 & 16,44 & 14,61 & 16,46 \\
\hline 13 & 9,69 & 12,55 & 9,68 & 12,54 & 9,66 & 12,55 \\
\hline
\end{tabular}

Para a potência reativa, os erros estão todos abaixo de $8,50 \%$, ressaltando mais uma vez que os resultados de Miranda et alli. (1990) correspondem ao valor da função de pertinência igual a 1 enquanto que para a função sinusoidal 0,5 .

Podem ser vistos através das tabelas 12 e 13, alguns valores de fluxos de potência ativa e respectivos erros.

Tabela 12: Fluxo Ativo (MW)

\begin{tabular}{|c|c|c|c|c|c|c|}
\hline Linha & \multicolumn{2}{|c|}{ FCN } & \multicolumn{2}{c|}{ FCNF } & \multicolumn{2}{c|}{ FCNTrap } \\
\hline Ni-Nf & $P_{k m}^{\text {min }}$ & $P_{k m}^{\max }$ & $P_{k m}^{m-\alpha}$ & $P_{k m}^{m+\alpha}$ & $P_{k m}^{P 2}$ & $P_{k m}^{P 3}$ \\
\hline 13 & 79,45 & 86,84 & 79,59 & 86,68 & 78,38 & 87,89 \\
\hline 412 & 40,17 & 44,39 & 40,05 & 44,50 & 40,12 & 44,44 \\
\hline 1617 & 2,677 & 2,934 & 2,778 & 2,832 & 2,61 & 3,00 \\
\hline 1518 & 5,252 & 5,807 & 5,456 & 5,602 & 5,19 & 5,87 \\
\hline 1819 & 2,183 & 2,410 & 2,293 & 2,299 & 2,14 & 2,45 \\
\hline 2527 & $-4,75$ & $-5,32$ & $-5,06$ & $-5,01$ & $-5,40$ & $-4,67$ \\
\hline 828 & $-0,63$ & $-0,47$ & $-0,63$ & $-0,47$ & $-0,61$ & $-0,48$ \\
\hline 628 & 18,06 & 19,84 & 18,15 & 19,75 & 17,91 & 19,99 \\
\hline
\end{tabular}

As tabelas 14 e 15 apresentam resultados para alguns fluxos reativos e respectivos erros. 
Tabela 13: Erros (\%) para Fluxos Ativos

\begin{tabular}{|c|c|c|c|c|}
\hline Linha & \multicolumn{2}{|c|}{ FCNF } & \multicolumn{2}{c|}{ FCNTrap } \\
\hline Ni-Nf & $P_{k m}^{m-\alpha}$ & $P_{k m}^{m+\alpha}$ & $P_{k m}^{P 2}$ & $P_{k m}^{P 3}$ \\
\hline 13 & $-0,18$ & 0,181 & 1,34 & $-1,21$ \\
\hline 412 & 0,286 & $-0,25$ & 0,12 & $-0,10$ \\
\hline 1617 & $-3,80$ & 3,470 & 2,33 & $-2,13$ \\
\hline 1518 & $-3,88$ & 3,517 & 1,28 & $-1,15$ \\
\hline 1819 & $-5,07$ & 4,594 & 1,86 & $-1,68$ \\
\hline 2527 & $-6,57$ & 5,845 & 1,64 & $-1,59$ \\
\hline 828 & $-0,5$ & 0,456 & 2,75 & $-3,94$ \\
\hline 628 & $-3,87$ & 3,540 & 0,82 & $-0,74$ \\
\hline
\end{tabular}

Tabela 14: Fluxo Reativo (MVAr)

\begin{tabular}{|c|c|c|c|c|c|c|}
\hline Linha & \multicolumn{2}{|c|}{ FCN } & \multicolumn{2}{c|}{ FCNF } & \multicolumn{2}{c|}{ FCNTrap } \\
\hline Ni-Nf & $Q_{k m}^{\text {min }}$ & $Q_{k m}^{\text {max }}$ & $Q_{k m}^{m-\alpha}$ & $Q_{k m}^{m+\alpha}$ & $Q_{k m}^{P 2}$ & $Q_{k m}^{P 3}$ \\
\hline 13 & 4,58 & 5,22 & 4,57 & 5,21 & 4,61 & 5,14 \\
\hline 412 & 13,32 & 14,07 & 13,62 & 13,77 & 13,33 & 14,04 \\
\hline 1617 & 1,02 & 1,36 & 1,02 & 1,37 & 1,04 & 1,34 \\
\hline 1518 & 1,47 & 1,75 & 1,46 & 1,76 & 1,50 & 1,72 \\
\hline 1819 & 0,55 & 0,73 & 0,55 & 0,74 & 0,57 & 0,71 \\
\hline 2527 & $-0,30$ & $-0,65$ & $-0,29$ & $-0,65$ & $-0,35$ & $-0,59$ \\
\hline 828 & $-3,05$ & $-1,91$ & $-3,00$ & $-1,97$ & $-3,08$ & $-1,89$ \\
\hline 628 & $-9,73$ & $-9,68$ & $-9,71$ & $-9,70$ & $-9,75$ & $-9,66$ \\
\hline
\end{tabular}

Tabela 15: Erros (\%) para Fluxos Reativos

\begin{tabular}{|c|c|c|c|c|}
\hline Linha & \multicolumn{2}{|c|}{ FCNF } & \multicolumn{2}{c|}{ FCNTrap } \\
\hline Ni-Nf & $Q_{k m}^{m-\alpha}$ & $Q_{k m}^{m+\alpha}$ & $Q_{k m}^{P 2}$ & $Q_{k m}^{P 3}$ \\
\hline 13 & 0,24 & 0,18 & $-0,67$ & 1,42 \\
\hline 412 & $-2,29$ & 2,17 & $-0,12$ & 0,21 \\
\hline 1617 & 0,73 & $-0,43$ & $-2,01$ & 1,64 \\
\hline 1518 & 0,73 & $-0,55$ & $-1,95$ & 1,70 \\
\hline 1819 & 0,96 & $-0,64$ & $-3,02$ & 2,38 \\
\hline 2527 & 1,82 & $-1,01$ & $-18,8$ & 8,45 \\
\hline 828 & 1,71 & $-3,41$ & $-0,94$ & 0,84 \\
\hline 628 & 0,18 & $-0,22$ & $-0,29$ & 0,24 \\
\hline
\end{tabular}

Na tabela 16 tem-se as perdas ativas nessas ligações e na tabela 17 os respectivos erros.

Tabela 16: Perdas (MW) na Transmissão

\begin{tabular}{|c|c|c|c|c|c|c|}
\hline Barra & \multicolumn{2}{|c|}{ FCN } & \multicolumn{2}{|c|}{ FCNF } & \multicolumn{2}{c|}{ FCNTrap } \\
\hline $\mathrm{N}^{\circ}$ & $P_{m i n}^{P e r}$ & $P_{m a x}^{P e r}$ & $P_{m-\alpha}^{P e r}$ & $P_{m+\alpha}^{P e r}$ & $P_{P 2}^{P e r}$ & $P_{P 3}^{P e r}$ \\
\hline 13 & 2,558 & 3,056 & 2,559 & 3,054 & 2,715 & 2,887 \\
\hline 412 & 0,000 & 0,000 & 0,000 & 0,000 & $-0,06$ & 0,062 \\
\hline 1617 & 0,006 & 0,008 & 0,006 & 0,008 & $-0,01$ & 0,023 \\
\hline 1518 & 0,030 & 0,037 & 0,029 & 0,037 & 0,016 & 0,050 \\
\hline 1819 & 0,003 & 0,004 & 0,003 & 0,004 & $-0,007$ & 0,013 \\
\hline 2527 & 0,024 & 0,030 & 0,024 & 0,030 & 0,006 & 0,048 \\
\hline 828 & 0,0007 & 0,0002 & 0,0003 & 0,0002 & $-0,009$ & 0,009 \\
\hline 628 & 0,055 & 0,067 & 0,055 & 0,067 & 0,054 & 0,068 \\
\hline
\end{tabular}

Como pode ser constatado na tabela 16, os resultados para o fluxo de carga proposto em Miranda et alli. (1990) contemplam valores negativos para as perdas na transmissão, o que é ressaltado no mesmo artigo, enquanto que para a metodologia aqui proposta, isto não
Tabela 17: Erros (\%) para Perdas Ativas

\begin{tabular}{|c|c|c|c|c|}
\hline Barra & \multicolumn{2}{|c|}{ FCNF } & \multicolumn{2}{c|}{ FCNTrap } \\
\hline $\mathrm{N}^{o}$ & $P_{m-\alpha}^{P e r}$ & $P_{m+\alpha}^{P e r}$ & $P_{P 2}^{P e r}$ & $P_{P 3}^{P e r}$ \\
\hline 13 & $-0,02$ & 0,069 & $-6,1$ & 5,5 \\
\hline 412 & 0 & 0 & - & - \\
\hline 1617 & 1,613 & 0 & 246,1 & -192 \\
\hline 1518 & 0,678 & 0,271 & 46,4 & $-36,2$ \\
\hline 1819 & 0 & 0 & 318,9 & -244 \\
\hline 2527 & 0,424 & 0 & 74,7 & $-57,4$ \\
\hline 828 & 57,14 & 0 & 1222 & 4486 \\
\hline 628 & 0 & 0 & 2,6 & $-1,8$ \\
\hline
\end{tabular}

Tabela 18: Quantidade de Flops

\begin{tabular}{|c|c|c|}
\hline FCN & FCNF & FCNTrap \\
\hline 3.009 .189 & 1.463 .066 & 2.132 .716 \\
\hline
\end{tabular}

ocorre e os maiores erros ocorrem nas linhas onde as perdas são muito baixas, como por exemplo na linha 8-28.

\section{COMENTÁRIOS E CONCLUSÕES}

Os algoritmos de fluxo de carga foram programados seguindo uma mesma metodologia, com diferenças apenas nas partes que contemplam os cálculos nebulosos.

A tabela 18 apresenta as respectivas quantidades de flops, obtidos com o MatLab` ${ }^{\circledR}$, para o sistema IEEE30.

A quantidade de flops para o FCN corresponde ao FCN aplicado nos pontos $m$ - $\alpha, m$ e $m+\alpha$. O FCNF apresenta menor quantidade de flops porque não utiliza a metodologia de "alfa cortes" (Pedrycz, W. \& Gomide, F., 1998).

O FCLF mostrou ótimos resultados, permitindo que técnicas de análise para o planejamento possam ser implementadas com boa precisão.

O FCNF proporciona bons resultados e em muitos casos melhores que os obtidos em Miranda et alli. (1990) e, ainda, solucionou o problema das perdas negativas.

A representação dos números nebulosos através da equação (1), além de gerar resultados com boa precisão e menor quantidade de flops, ainda permite ampliar a análise dos resultados, uma vez que entre os pontos de mínima e máxima potência, a função de pertinência apresenta valores contidos no intervalo $[0,5 ; 1]$, enquanto que para a função trapezoidal a pertinência é sempre unitária. A função sinusoidal permite aplicações que traduzem os resultados numéricos em lingüísticos, como exemplifica a figura 3 . 


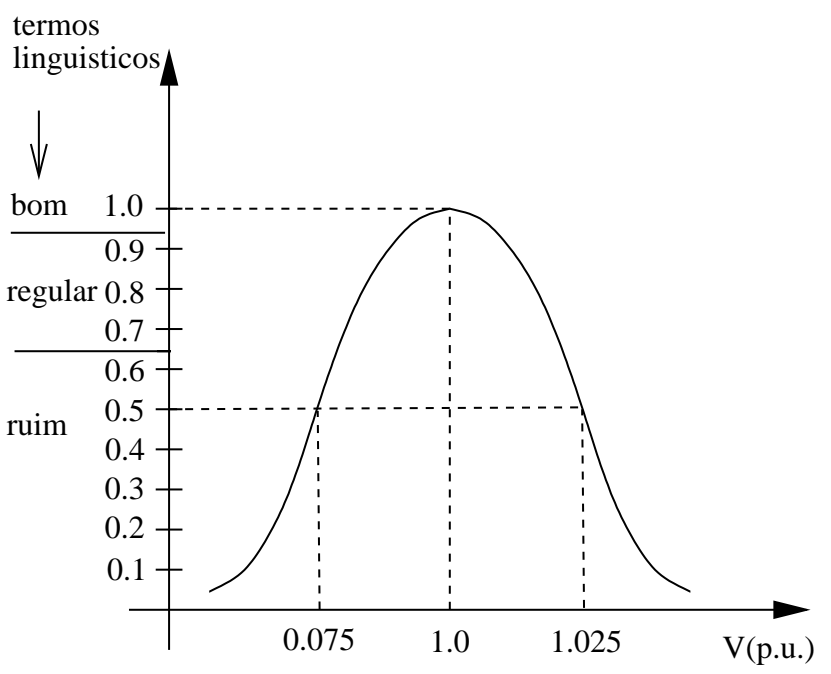

Figura 3: Exemplo de interpretação de resultados

\section{APÊNDICE A}

Foi necessária a definição de uma nova função de multiplicação para ser utilizada nos cálculos dos fluxos, porque a definida em Pereira, M. A. \& Murari, C. A. F. (1999) não estava proporcionando os resultados esperados.

Como na matemática nebulosa deve-se sempre escolher as melhores funções e operações, em função do tipo de aplicação com o qual se está trabalhando, definiu-se a seguinte função:

$$
\begin{aligned}
& A\left(m_{A}, \alpha_{A}\right)=X \underline{\otimes} Y \\
& m_{A}=m_{X} * m_{Y} \\
& \alpha_{A}=\alpha_{\text {ord }}(2) ;
\end{aligned}
$$

onde $\alpha_{\text {ord }}(2)$ é o segundo elemento do vetor:

$$
\begin{aligned}
& \alpha_{\text {ord }}=\operatorname{str}\left(\left|m_{A}-\left[\left(m_{X}-\alpha_{X}\right) *\left(m_{Y}-\alpha_{Y}\right)\right]\right|,\right. \\
& \left|m_{A}-\left[\left(m_{X}-\alpha_{X}\right) *\left(m_{Y}+\alpha_{Y}\right)\right]\right|, \\
& \left|m_{A}-\left[\left(m_{X}+\alpha_{X}\right) *\left(m_{Y}-\alpha_{Y}\right)\right]\right|, \\
& \left.\left|m_{A}-\left[\left(m_{X}+\alpha_{X}\right) *\left(m_{Y}+\alpha_{Y}\right)\right]\right|\right)
\end{aligned}
$$

e str é uma rotina que coloca os elementos em ordem crescente.

\section{APÊNDICE B}

Caso fossem utilizadas as funções seno e cosseno convencionais nos cálculos nebulosos, os resultados ficariam a desejar, e assim sendo, estabeleceu-se uma formulação para efetuar o cálculo destas operações de forma nebulosa (ressalta-se que estes cálculos são uma aproximação de um seno ou cosseno fuzzy).
Seno fuzzy:

$$
\begin{aligned}
& \sin f u z z y\left(m_{\theta}, \alpha_{\theta}\right)=\left(m_{\sin \theta}, \alpha_{\sin \theta}\right) \\
& m_{\sin \theta}=\left(\sin \left(m_{\theta}-\alpha_{\theta}\right)+\sin \left(m_{\theta}+\alpha_{\theta}\right)\right) / 2 \\
& \alpha_{\sin \theta}=\left(\left|\left(\left|m_{\sin \theta}\right|-\left|\sin \left(m_{\theta}-\alpha_{\theta}\right)\right|\right)\right|+\right. \\
& \left.\quad\left|\left(\left|m_{\sin \theta}\right|+\left|\sin \left(m_{\theta}+\alpha_{\theta}\right)\right|\right)\right|\right) / 2
\end{aligned}
$$

Cosseno fuzzy:

$$
\begin{aligned}
& \cos f u z z y\left(m_{\theta}, \alpha_{\theta}\right)=\left(m_{\cos \theta}, \alpha_{\cos \theta}\right) \\
& m_{\cos \theta}=\left(\cos \left(m_{\theta}-\alpha_{\theta}\right)+\cos \left(m_{\theta}+\alpha_{\theta}\right)\right) / 2 \\
& \alpha_{\cos \theta}=\left(\left|\left(\left|m_{\cos \theta}\right|-\left|\cos \left(m_{\theta}-\alpha_{\theta}\right)\right|\right)\right|+\right. \\
& \left.\quad\left|\left(\left|m_{\cos \theta}\right|+\left|\cos \left(m_{\theta}+\alpha_{\theta}\right)\right|\right)\right|\right) / 2
\end{aligned}
$$

\section{REFERÊNCIAS}

Freris, L.L. \& Sasson, A.M. (1968). Investigation of the load-flow problem, Proceedings of IEE, Vol. 115 (10), pp. 1459-1470.

Kenarangui, R. \& Seifi, A. (1994). Fuzzy power flow analysis, Electric power systems research, 29: pp. 105-109.

Meliopoulos, A.P S., Cokkinides, G.J. \& Chao, X.Y. (1990). A new probabilistic power flow analysis method, IEE Transactions on Power Systems, Vol 5 (1), pp. 182-189.

Miranda, V., Matos, M.A.A.C. \& Saraiva, J.T. (1990). Fuzzy load flow - New algorithms incorporating uncertain generetion and load representation, Proceedings of the Tenth Power systems Computation Conferece, Graz, Austria, pp. 621-627.

Monticelli, A. (1983). Fluxo de carga em redes de energia elétrica. Edgard Blücher, São Paulo, Brasil.

Pedrycz, W. \& Gomide, F. (1998). An introduction to fuzzy sets: Analysis and design. A Bradford Book - The MIT Press.

Pereira, M.A. \& Murari, C.A.F. (1999). Electrical distribution system fuzzy load estimation, ISAP'99, Rio de Janeiro, pp. 370-375.

Zadeh, L.A. (1965). Fuzzy sets, Information and Control, Vol. 8, New York, pp. 338-353.

Zadeh, L.A. (1978). Fuzzy sets as a basis for a theory of possibility, Fuzzy Sets and Systems, 1: pp. 3-28. 